\title{
Geodata collection and visualisation in orchards: interfacing science-grower data using a disease example (European canker in apple, Neonectria ditissima)
}

\author{
Juliane Bührdel ${ }^{1}$, Monika Walter ${ }^{2}$ and Rebecca E. Campbell2,* \\ ${ }^{1}$ HTW Dresden University of Applied Sciences, Dresden, Germany \\ ${ }^{2}$ The New Zealand Institute for Plant and Food Research Limited, Old Mill Rd, RD3, Motueka 7198, New Zealand \\ *Corresponding author: Rebecca.campbell@plantandfood.co.nz
}

(Original submission received 10 August 2020; accepted in revised form 26 November 2020)

\begin{abstract}
The collection and visualisation of data in orchards are important for management of many orchard processes, including pests and diseases. We present methods combining visualising data with efficient, accurate, standardised data collection, using European canker in apple orchards as an exemplar. Using growercollected current and historical disease data, we investigated Environmental Systems Research Institute (ESRI) ArcGIS tools to analyse and visualise data. Historical data were collected by growers on paper and current data, also collected by growers, using Survey123. ArcGIS Pro was the operating software for analysis, and ArcGIS Online, Web Maps and ArcGIS Dashboards, for visualisation. Data collection, summarising and visualisation were more efficient using Survey123, than paper collection and subsequent data entry. Higher quality data, including spatial location of the disease, informed disease patterns. A standardised geodatabase enabled efficient data querying and analytics to understand disease distribution and temporal dynamics. This study exemplars a standardised disease and pest database to benefit both scientific and industry data management. Geodata collection, combined with visualisation, facilitates the use of data to understand disease and pest dynamics. These techniques offer opportunity for a cohesive industry approach to area-wide disease and pest monitoring and management, integrating previously disparate datasets by using location.
\end{abstract}

Keywords area-wide pest and disease management, dashboard, European canker, GIS, survey123, monitoring

\section{INTRODUCTION}

Digital tools and information technologies are increasingly making data readily available to support management decisions for crop protection (Padgitt et al. 2001, National Research Council USA 1997, Liu et al. 2017, Oliver 2010). These tools enable site-specific, localised crop monitoring, including the recording and understanding of spatial and temporal variability (Cambouris et al. 2014), to improve the uptake of more sustainable or cost-effective practices e.g. precision agriculture (Padgitt et al. 2001, Oliver 2010). For pest and pathogen control, such tools can increase the efficiency of monitoring crop health and obtain data that could be used to inform targeted agrichemical spraying or targeted disease control. The collection and spatial visualisation of pest and disease data in orchards are important steps for their effective management. Analyses of spatial patterns of pests and diseases are useful to assist both scientific research and crop management (Alspach \& Bus 1999, Rodriguez et al. 2009, Oliver 2010, Campbell et al. 2016). In the research of European canker (caused by Neonectria ditissima) in apple (Malus x domestica), the collection of canker data for an orchard is essential to help growers/companies to identify visual disease clusters/hot-spots (aggregation of disease records) that can then be targeted for more intense disease surveillance and management such as by ongoing inoculum removal (Campbell et al. 2016, Di Iorio et al. 2019). Currently New Zealand growers use several surveillance methods including recording disease incidence at the block, row or tree resolution (Di Iorio 2019, Di Iorio et al. 2019). The success of management efforts is directly reflected in the costs of disease control and plant removal (Liu et al. 2017).

One aspect of precision agriculture is georeferencing - associating measurements with specific georeferenced locations (Oliver 2010, Padgitt et al. 2001). The resulting spatial visualisation and analysis enables better understanding of spatial and temporal dynamics (Rodriguez et al. 2009, Orozco-Fuentes et al. 2018) than traditional data collection methods. Historically, data were collected manually in a paper notebook, with additional steps required to digitalise the data for any summaries and analyses. This would add to the time taken for data processing and multiple opportunities for potential mistakes (be they typing, reading or concentration related). Well-designed data collection apps can be used to collect the data directly in a digital format and load data immediately into visualisation tools, thus reducing the potential for errors (Luvisi et al. 2016, Malek et al. 2018, Srivastava et al. 2020, Warkentine 2020). 
A standardised data collection app can, for example, be restricted only to sensible standardised values and formats (e.g. string, number, etc.), without requiring additional resources to paper data collection in the field (Luvisi et al. 2016, ESRI 2020a).

Transitioning between traditional paper data collection and digitised spatial data is not always easily incorporated into everyday management. Therefore it is important that the method be easy to use, fit for purpose, evaluated for practicality, and ultimately, developed in consultation with the end-users (Padgitt et al. 2001, Malek et al. 2018). Furthermore, it needs to be compatible with the integration of historical data, while providing a clear standardised digital way to gather and record future data. There is a fine balance between collecting enough useful data to best inform science and management decisions, and the amount of effort or cost required in obtaining them. Finding methods to improve the expedition, accuracy, and standardisation of data collection increases the amount of useful information that can be obtained without increasing the time and resources involved (Ahmed et al. 2018).

Geospatial databases provide an opportunity to efficiently gather and store digital and spatial records. Storing the spatial data creates opportunities to understand simultaneous interacting factors in orchard blocks while also providing an understanding of the spatial nature of disease or pest spread (Oliver 2010). Furthermore, the tools to visualise spatial and temporal data efficiently provide growers and scientists with powerful exploratory options to understand their management efforts and formulate hypotheses and solutions to pest and disease management.

The objectives of this study were to: establish and trial a georeferenced digital disease data collection method in apple orchards; and to create a database as a platform for both interactive grower data analytics and, in the future, a resource for bio-protection science to aid understanding of disease spread and area-wide management.

\section{MATERIALS AND METHODS}

ArcGIS Pro (v2.4, ESRI) was used as the operating software for analysis and digitising. ArcGIS Online and associated services were used, including Web Maps and ArcGIS Dashboards for the visualisation and Survey123 for data collection (Figure 1). Two different data collection strategies already used in commercial apple orchards for European canker were explored: tree-level disease incidence and row-level disease incidence (Di Iorio 2019, Di Iorio et al. 2019). Feedback and trialling from commercial growers were sought and implemented in the design of the apps and dashboards.

\section{Digitised field data collection}

Two methods were used to create two types of Survey123 apps. Both create surveys for flexible use on a device (e.g. smartphone, computer, tablet), with or without field internet connection. For new data collection (which could later be manually integrated with historical data), the Web Designer option in Survey123 was used. For adding to existing and historical data (e.g. data already collected at the tree level),

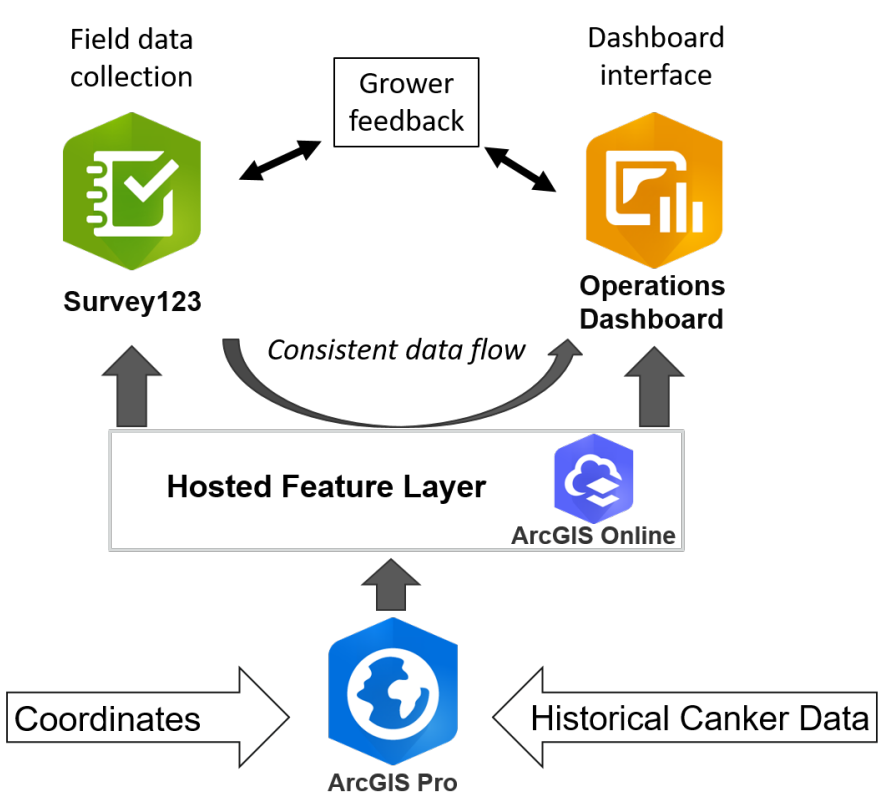

Figure 1 Workflow summary for linking existing disease incidence data with apps for in-field data collection, data management, data visualisation and interaction.

Survey123 Connect was used because it has wider smartform capabilities through Microsoft Excel ${ }^{\circledR}$ spreadsheets (XLSForm files), which were necessary to create a baseline of existing data to which to add new data. The data collected were stored in a table or Feature Layer and exported as either "CSV", Excel, Shapefile or File Geodatabase formats.

\section{Preparing and structuring existing data}

Two Microsoft Excel ${ }^{\circledR}$ tables (.csv-files) were used to structure the historical grower data of canker incidence. One table contained the coordinates of each tree; the other contained the canker data collected by the growers in previous years. These were 'joined' on the field of a unique 'tree ID' (ESRI 2020b). The tree ID is formed from the concatenation of block-row-bay-tree to create a unique TreeID (e.g. BlockA-1-3-2). Often existing grower canker data are recorded in spreadsheets at the 'row' level. In these cases the canker incidence for the row was joined to a digitised line feature. Therefore the position of the disease along the row dimension was unknown and 'hot-spots' (areas with high disease incidence) appeared as full-length rows. Thus a direct spatial comparison was not possible between new and existing data; however, data visualisation of summaries at row level was possible.

\section{Digitising individual trees: Python code}

Without a Global Navigation Satellite System (GNSS) receiver that has accuracy of $<1 \mathrm{~m}$, collecting tree-level location without specialist equipment is not currently possible or practical for scientists or growers. Therefore, a grid of tree coordinates was created using a Python (Van Rossum \& Drake Jr 1995) program, then these points were 'joined' to existing canker data, and future data collection was 
subsequently referenced to these points (Di Iorio 2019). A Python code referring to a template Excel sheet was used to create a table of all the coordinates for individual trees. Most blocks were regular rectangle/square shaped, therefore the Python code created single tree coordinates of a rectangle/ square-shaped block (custom-shaped blocks would require additional editing). The template Excel sheet contained the coordinates of the four corners of the block, the number of trees in a row, the number of rows, which corner to start the rows from (A, B, C or D; Figure 2) and the orientation of the rows (vertical or horizontal; Figure 2) (Di Iorio 2019). The code calculated vectors between the corner coordinates of the block, "cutting" them into the required number of rows and trees in a row and calculating the coordinates for each single point (tree location).

ArcGIS Pro was used to digitise the blocks of an orchard to obtain the corner coordinates for use in the input template for the Python code (Di Iorio 2019). With the Feature Layer then stored in the geodatabase, the block scale polygons can also be used for further block-scale spatial analyses when required (as relational features with the tree- or row-level data).

\section{Historical row-level data}

Data that were collected at the row-level were digitised as lines instead of points using the same Python code (Di Iorio 2019) to obtain the coordinates of each line. Instead of the 'number of trees', two points per row were used, the relevant row number being assigned to each point in the "csv" file and converted into a Feature Layer in ArcGIS Pro.

\section{App for row- and bay-level data collection (Survey123 Web designer)}

With the Web designer, a simple survey was created for the grower that stores the standard data they currently collect on paper e.g. Block, Row, Year, Walk (season), Canker presence or need for tree removal due to canker, and Location. Using the "GeoPoint" field to collect a location using the device's GNSS receiver ( $\pm 5-10 \mathrm{~m}$ accuracy) offered an improvement of spatial resolution over row-level data without extra effort. The growers could continue their row-level analyses, but could also obtain a location along the row (to the accuracy of the GNSS receiver used in their device) without having to count single trees. The Web designer created a new Feature Layer based on a survey that did not automatically connect to historical data which have been collected into a spreadsheet. Historical data had to be formatted and combined manually to visualise new and historical data together (joined by the 'row' ID), for example, in the dashboard.

\section{App for tree-level data collection based on an existing Feature Layer (Survey123 Connect)}

Survey123 Connect used existing (e.g. historically collected data) names and column types (e.g. numeric, text) based on an existing Feature Layer (spreadsheet) to create the survey questions. The "pulldata" (XLSForm) command automatically associates a point (tree) to spatial coordinates from an existing point (see 'Digitising individual trees'), saved in a separate csv-file and connected to Survey123, based on the same (row-bay-tree) ID. For example, the easiest way to record tree-level data (without a highly accurate GNSS receiver) is to use the created tree locations and connect them to the incoming data by their unique row-bay-tree combination.

\section{Grower interface: ArcGIS Dashboards}

The ArcGIS Dashboards on ArcGIS Online was used to create the grower interface for the summarised data and maps. A hosted Feature Layer was created in ArcGIS Online and used to create a Web Map and Dashboard. 'Editing and synchronisation' was enabled to keep the data visualisations up-to-date when data were deleted, changed or added (for example, with Survey123). The hosted Feature Layer was used for charts, calculations and indicators to show in a dashboard.

Two different examples of dashboards were created to suit the needs of different management practices and to customise the canker data summaries useful to the individual growers and their existing management systems. Visualisation graphs and statistics were created to match or improve on exploratory analyses that would normally be created by the growers in Excel each time canker monitoring is completed (in some cases 4 times per year). These analyses included charts (histograms, line graphs), indicator statistics (\% disease incidence), category selectors (year, season) and filters (number of 'strikes' (tree incidence), removals (full tree removal due to disease)). Interactive actions between the dashboard elements were added. For example, changes in the data selected (e.g. year) induced changes in the map and charts being visualised. These dashboards were based

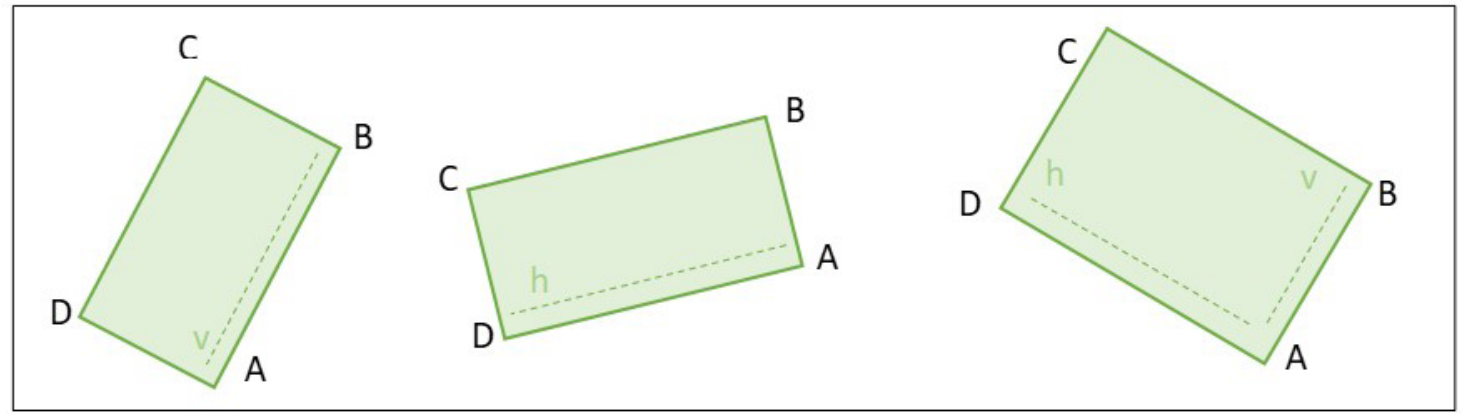

Figure 2 Block arrangement for corner reference points to create a grid of tree locations using Python code. ' $\mathrm{A}$ ' is always the point closest to the bottom right; the other corners are named anticlockwise and the orientation follows the rule: horizontal $(\mathrm{h})$ is parallel to DA and vertical $(\mathrm{v})$ is parallel to $\mathrm{AB}$. 
on the hosted Feature Layers with historical data (where available) and the data collected from Survey123. In this case, data can flow directly and continuously because both the Survey 123 form as well as the dashboard are based on the same hosted Feature Layer.

\section{On-orchard trials}

Grower feedback was sought with one to one on orchard meetings with individual growers at the initiation of the project to gauge interest, potential and for the grower to share the limitations of their existing methods, which could be improved. Grower feedback was also sought in the same way and incorporated to test, improve and implement the data collection app, the utility of the dashboards and which data growers wanted visualised and summarised expeditiously. Two growers were shown Surveys and Dashboards created from either their existing disease data or sample data to illustrate possibilities with other elements of the software (e.g. improved location data). The demonstration examples were created for two blocks and 13 blocks for the two growers respectively.

\section{RESULTS}

Two exemplar data-collection survey apps were created for two separate case studies: one for tree-level data collection, to align with existing digitised trees (tree level accuracy); and the other for entering data at the row-level and using the device GNSS for location along the row $( \pm 5-10 \mathrm{~m}$, approximately bay-level). The interface and data entry were made as simple as possible and pre-populated defaults or 'domains' were used where practical, to ensure the least amount of manual data entry (Figure 3).

\section{Grower needs and app customisation}

\section{Case study 1}

The first case-study example dashboard (Figure 4) shows five different elements: a Category Selector (year); a Legend; an Indicator (summary \%); a Map; and a Serial Chart. The focus of these data are the map of individual tree-level data to identify changing or persistent visual hot-spots over time, along with an overall summary of percent incidence of canker for the block.

For the first case study, the orchard had relatively low canker incidence (around 2-4\%) and recorded tree-level data by painting the trunks of the trees with different colours corresponding to the year in which they expressed canker. There were no paper recordings of tree-level canker except where The New Zealand Institute for Plant and Food Research Limited had recorded these data for research purposes. The grower understood broadly where their hot-spots (areas of high disease incidence) were, but was interested in knowing how areas of disease expand or contract or when new locations start to appear before they develop into obvious hot-spots. The app requirements in this case study were aligned to the grower's current twostep management practice of initial recording followed by removal if required. This grower preferred the ability to log

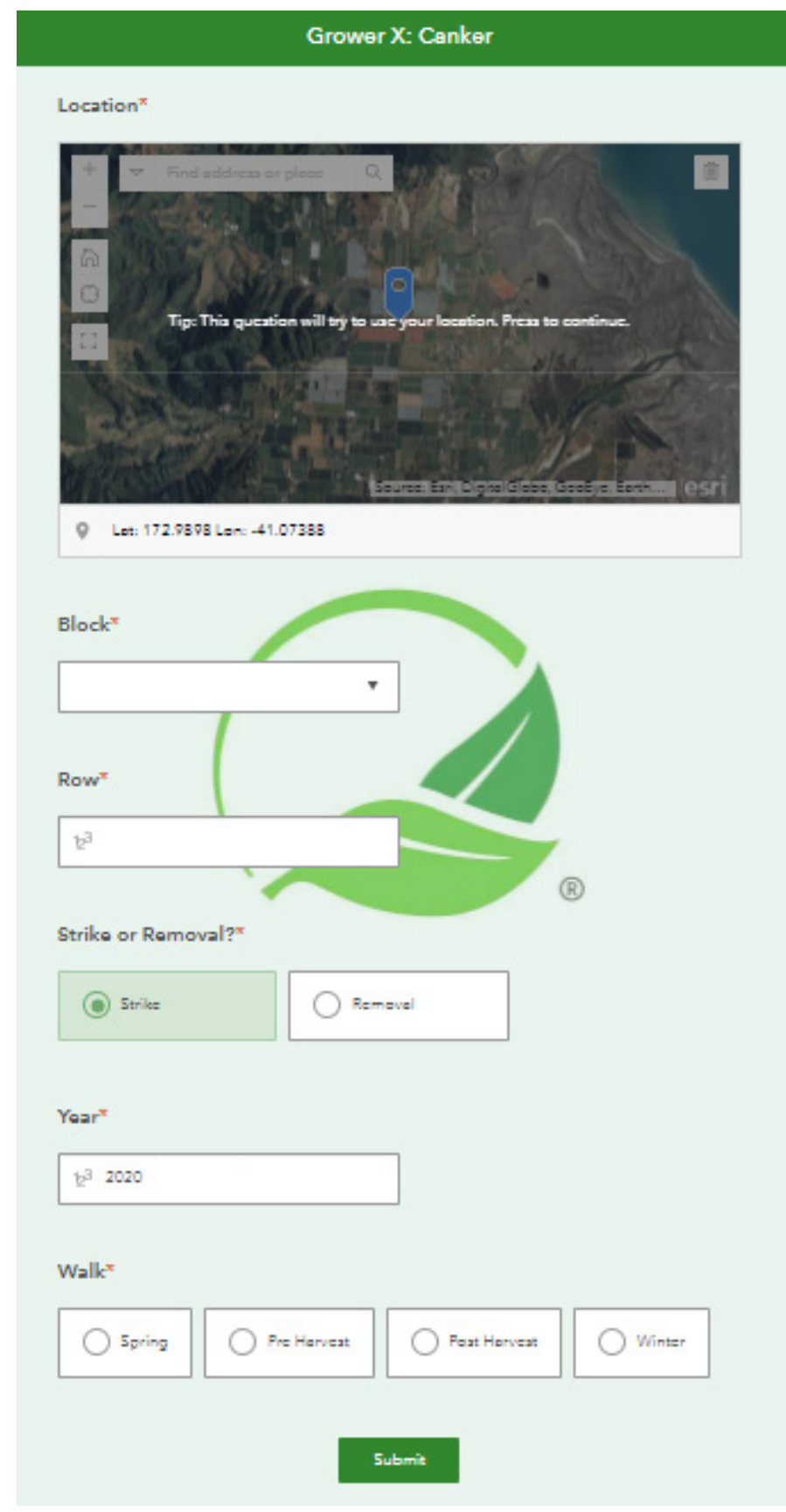

Figure 3 App view example for collecting European canker disease data with a GNSS location for each recorded disease incidence (device receiver accuracy in this case approximated bay level). Fields are pre-populated or have a restricted domain to make field data entry as minimal as possible.

the canker initially at a row or approximate part of the row level and then have a second user update the data in the app with the removal information. These removals would have more specific details such as type of wound infection, how much of the tree/branch was cut, whether the trunk needed canker removal, and to be updatable when it was deemed not to be canker caused by $N$. ditissima. 


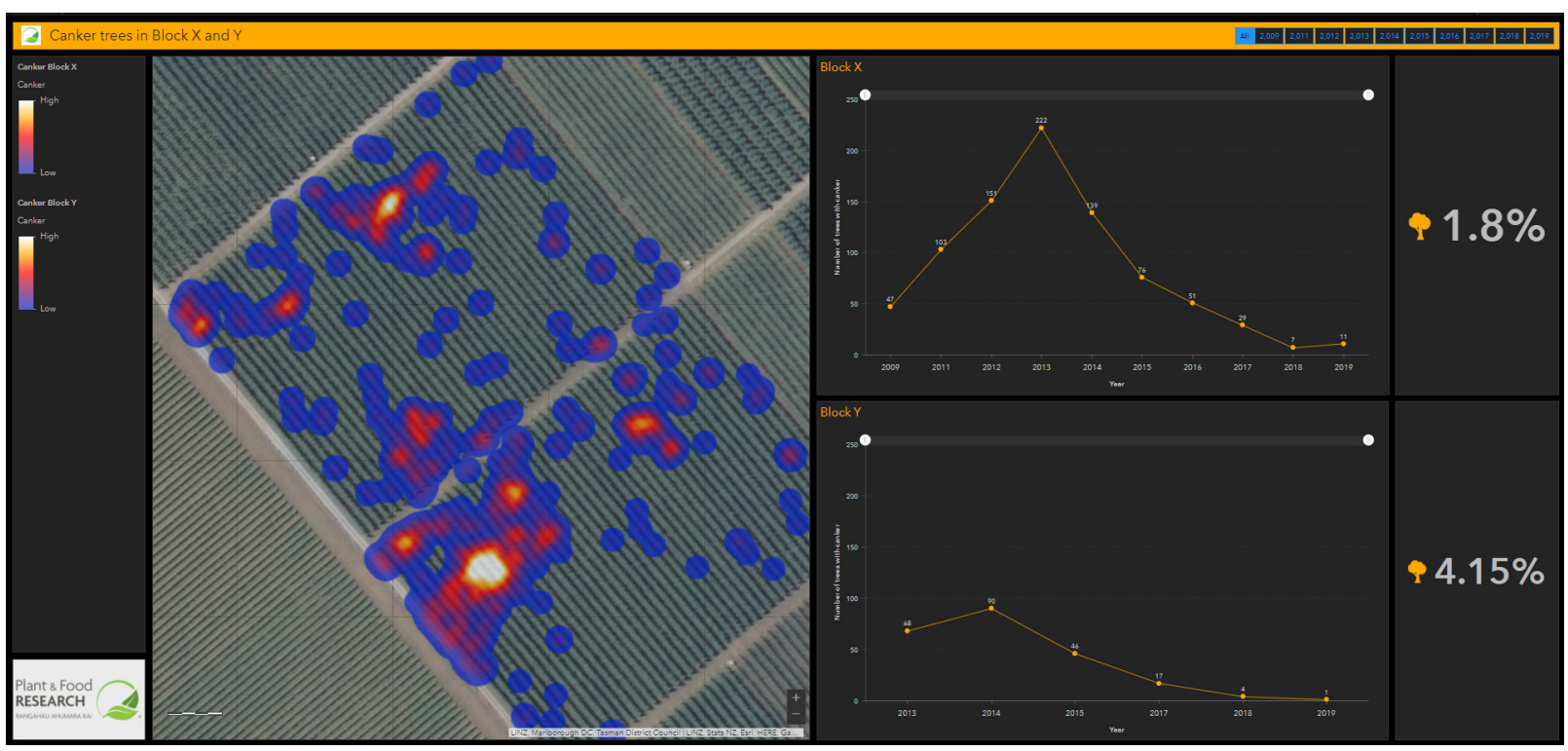

Figure 4 Example dashboard showing European canker incidence density as a heat map, total numbers of trees with canker selectable by each year and the overall block percentages of tree incidence of canker.

\section{Case study 2}

A different data-presentation style was trialled for the second case-study dashboard (Figure 5). In this case, the grower wanted an emphasis on tree removals and disease incidence at the row level. This case study separated the data into seasonal (rather than yearly) summaries to aid management. In this example, reporting the number of 'strikes' (disease incidence on a tree) and removals (full tree removal due to disease) was more important than the specific location/map. However, after implementing the data-collection app in this orchard, the managers saw potential value in receiving a map summary as well, and in summarising at the more detailed spatial scale of $\pm 5-10 \mathrm{~m}$, which can be achieved with current standard technology in a smartphone.
The canker strategy for the second grower case study involved primarily one individual in charge of simultaneously recording and removing canker throughout each block. Data entry was through one device and needed to be as simple and quick as possible. Therefore, the app was customised differently to case study 1 . Some blocks had over $50 \%$ disease incidence, so any efficiencies in data entry were important. Historically, this grower recorded only at the row level, and did not wish to significantly increase the time involved in recording at this resolution. Therefore the app was customised to autofill all answers apart from tapping to record the location (accuracy of $\pm 5-10 \mathrm{~m}$ ) and entering the row number. Canker records were entered as either a 'strike' or a tree 'removal' and were able to be summarised by year, season, management area or block. The manager

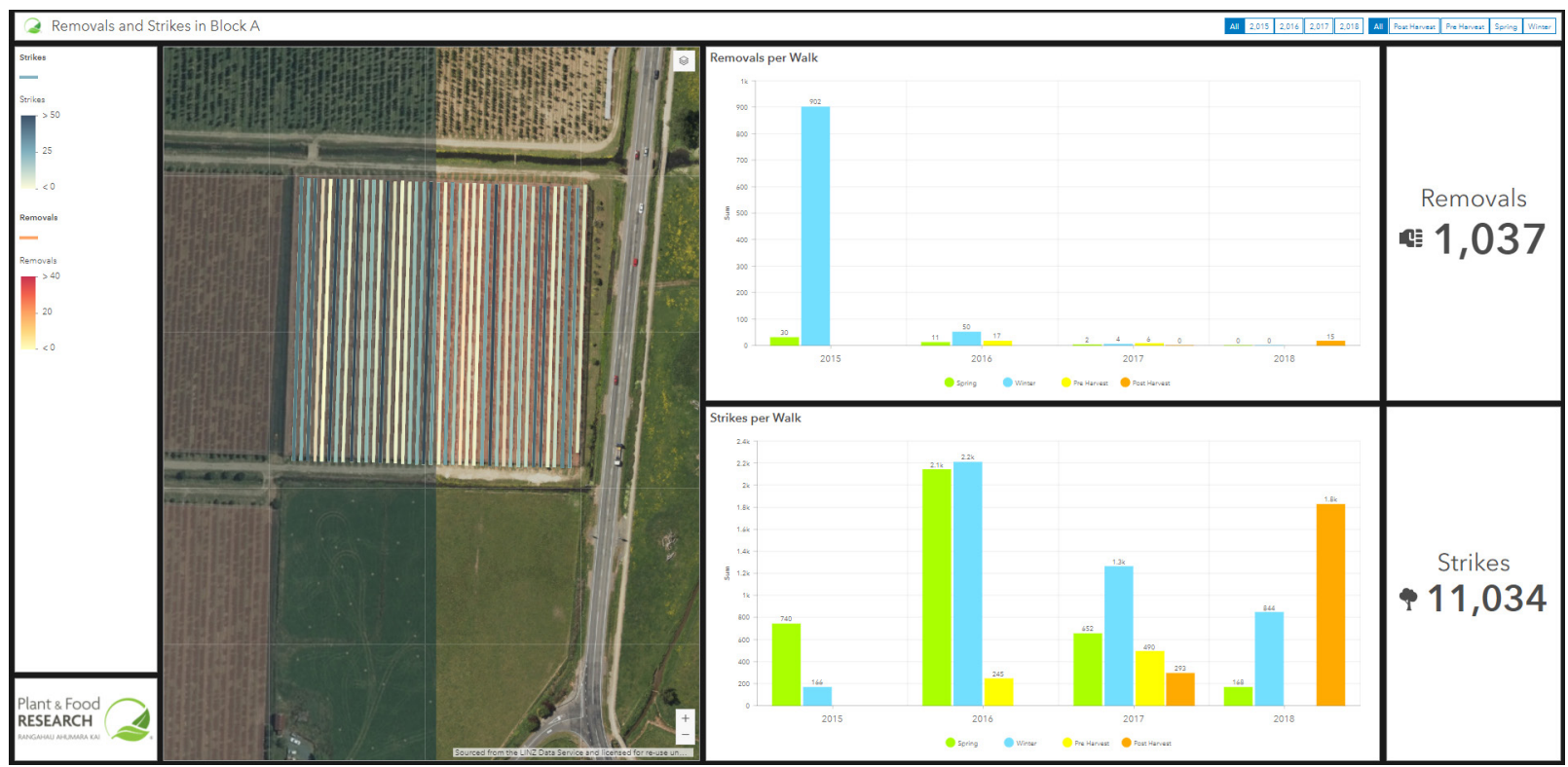

Figure 5 Dashboard example where row and block level data are presented. 'Strikes' (European canker disease incidence) and 'removals' (whole-tree removals due to disease) were of interest, as were the year and time of year (season) in which the canker was removed. 
emphasised that knowing quickly and accurately how many removals, where and when, would be important to understanding the costs incurred in managing canker.

\section{DISCUSSION}

In this project, we worked with apple growers to understand their needs in recording and managing European canker. As a result of this engagement, we were able to provide them with the ability to customise data collection and visualisation to fit their specific management needs. At the same time, we provided a platform for standardised efficient data collection and storage, with the ability to create a standardised database in the future.

By improving the data-collection process expeditiously (from paper data collection to digital), we increased the amount and quality of useful information recorded without increasing the time and resources involved. Steps of paper data collection, data entry and drawing smmary figures were removed, and a change from writing on paper, to tapping a pre-populated screen survey were minor adjustments to existing practices. It was critical to find a balance between the trade-offs of useful quantity and quality of information for orchard management on one hand and the degree of effort or cost required to obtain these data on the other. For example, tree-level data can show where actual hotspots (areas of high disease density) are within an orchard and how pests and diseases spread over time (Alspach et al. 1999, Campbell et al. 2016), but such data require more collection effort than row-level disease and pest recording when using the traditional 'paper and pencil' methods. The digital data collection app and dashboard developed and applied in the current study, increased data collection efficiency and decreased the risk of data entry error by automatically visualising and summarising entered data without further processing. This technology has thus enabled implementation of information technologies to bring data together to inform decisions for crop protection, and also enabled improvement of management strategies (National Research Council USA 1997, Liu et al. 2017).

Optimal data collection will be paramount to enable future precision horticulture technologies such as targeted spraying, provenance tracking and also to ensure best practice for pest and disease management (Oliver 2010). Accurate, reliable, easy data collection will have value for growers, as they can use this information for the purpose of better management practices or, where appropriate, understanding the science behind disease control. Furthermore, it is increasingly important to reduce costs and target management efficiencies, and these data and accessible outputs will empower growers to achieve these goals effectively. It would be beneficial to quantify the costs and benefits of our new proposed digital data collection and visualisation, compared to current practices, with future work. To improve adoption, a quantified economic and statistical analysis of efficacy in management practices, including time and cost would be beneficial to understand what gains can be achieved using a simple app. Anecdotally, the methods trialled in this study have improved the grower understanding of disease distribution and given them more confidence in targeting hotspots with appropriate management responses.

In the past, pest and disease data have sometimes been collected in an ad hoc fashion and stored inefficiently, or not at all; therefore, the full value from the data-collection effort has not been gained. Furthermore, spatial location has been difficult to capture and store; for example, European canker data are often no more than row-level records because of the time required for tree-level data collection and data management (on paper). Row- and block-level summaries can be sufficient to determine whether management practices are effective in controlling the overall rate of disease or whether general disease incidence is increasing or decreasing (Campbell et al. 2016, Di Iorio et al. 2019). However, these data cannot indicate whether the disease is concentrated at one end of a row or distributed throughout the row (Di Iorio 2019). Such information is desirable to determine hot-spots (statistical or visual) or sources of infection (e.g. neighbouring land use). The ability to record location, even at the currently available $\pm 5-10 \mathrm{~m}$ resolution, greatly enhances the ability to target problematic areas of the orchard, particularly when hot-spots are across rows (not aligned with rows). The resolution of tree-level data has advantages for understanding repeat-infection rates and tree history, thus adding confidence and guidance to costly tree removal. Additionally, collection of quality standardised spatial data can allow statistical comparisons, quantify growth and retraction of hot-spots and facilitate retention of knowledge of historic hot-spots especially with changes in staff over time.

Grower feedback was crucial to adapting methods that would be embraced by the growers themselves (Padgitt et al. 2001, Santiago-Brown 2014). At the orchard-wide spatial scale needed, growers need to be empowered to collect, visualise and understand the implications of these data. Maps and interactive dashboards are ideal for this communication connection between science and orchard management (Monmonier 1996, Benge et al. 2014).

The growers involved in this study showed strong positive interest because their current disease data collection practices are based on paper notes with a manager subsequently entering and manually summarising the data. Some have been exploring different tools (apps, software) for other types of data collection and analysis (e.g. crop load, thinning, payroll); however, they were concerned that apps that try to cover a large range of purposes tend to be more complicated and less streamlined or realistic for simple, efficient, specialised disease data management. No other tools are yet available for them simply to collect their disease data digitally and automatically summarise and visualise outcomes, to simplify management decisions.

The one-on-one meetings with the growers also showed that the uses and needs differed among the different growers surveyed, specific to their management practices. For example, some growers preferred row-level data, whereas others preferred tree-level data. Because of the flexibility to create and customise the surveys and the dashboards, it was possible to meet the various growers' needs.

Two grower-science data interfaces were tested with two different growers and are flexible enough to customise and 
integrate grower feedback to obtain a better balance between data collection, visualisation and resources. It is important to make the collection (and visualisation) as standardised as possible (Srivastava et al. 2020) to enable optimal and future data use, both for the overview and for analysis within larger companies and across regions in the interest of biosecurity and area-wide bioprotection (Aketarawong et al. 2011). Data custody and management can also be challenging, and this platform allows a standardised geodatabase which automatically maintains the data quality and interactive interface. Thus, these growers have disease data in the form of a standardised database of georeferenced disease incidence. These data have multiple future uses for orchard management, science and bioprotection (including biosecurity) (Sayers et al. 2013). One factor which may need adressing, is that an increase in quantity of quality data may require staff with skills in big data management, however this could be an opportunity rather than a disadvantage of these methods.

The GIS and ESRI products have support and continual development and are used for many diverse applications (Zhu 2016, Jordan et al. 2019, Warkentine 2020). For our purpose, ESRI products were effective because they are intuitive, customisable and connectable for a continuous data flow. The flexibility not explored in this study includes the use of barcodes/QR codes in the data collection, which could assist with location and interaction with other grower data (e.g. picking bin data). However, with technology improving all the time, the most promising advances will be the ability to link data by location to $<1 \mathrm{~m}$ accuracy with everyday GNSS receivers in devices such as smartphones. The current accuracy of a smartphone GNSS receiver is $\pm 5-10 \mathrm{~m}$, which is sufficient to determine which part of the row (an improvement on row only records), and, in conjunction with entering the row number, approximates bay resolution. However, \pm 5-10 m resolutionfor orchard management is insufficient to recognise an accurate location of an individual tree or distinguish between rows (when row number not entered). Promisingly, GNSS receivers in smartphones/devices are expected to achieve location accuracy to $<1 \mathrm{~m}$ by 2023 (Cozzens 2020); this will enable tree-level data records without extra effort (row, tree data entry) or specialist devices.

We do acknowledge there are several other software packages available for facilitating orchard management; however, most are targeted to manage payroll, and none is scientifically developed to inform disease management specifically. While some growers will prefer one app to cover many applications, others find it easier and more fit-forpurpose to use an app customised in development with the growers' specific need(s). The data collected with methods described in this study can be spatially integrated with data from other software, and can be exported as spreadsheets or shapefiles in a number of versatile formats for future use.

\section{CONCLUSIONS}

This study describes the successful implementation of simple data collection apps and dashboards for visualisation of disease incidence in orchards. These can be run as stand- alone or incorporated with other orchard mapping tools. These apps improved the ease, accuracy and efficiency of data collection and processing, thus providing customisable tools for utilising pest and disease information for applied orchard management.

\section{ACKNOWLEDGEMENTS}

This research would not have been possible without the support from the orchard growers, managers and field staff. This research was funded by The New Zealand Institute for Plant and Food Research Limited's Strategic Science Investment Fund (SSIF). We also thank colleagues Lloyd Stringer and Rebecca Woolley for their constructive feedback.

\section{REFERENCES}

Ahmed R, Robinson R, Elsony A, Thomson R, Squire SB, Malmborg R, Burney P, Mortimer K 2018. A comparison of smartphone and paper datacollection tools in the Burden of Obstructive Lung Disease (BOLD) study in Gezira state, Sudan. PLoS ONE 13(3): e0193917. https:// doi.org/10.1371/journal.pone.0193917

Aketarawong N, Chinvinijkul S, Orankanok W, Guglielmino CR, Franz G, Malacrida AR, Thanaphum S 2010. The utility of microsatellite DNA markers for the evaluation of area-wide integrated pest management using SIT for the fruit fly, Bactrocera dorsalis (Hendel), control programs in Thailand. Genetica 139: 129-140. https:// doi.org/10.1007/s10709-010-9510-8

Alspach P , Bus VGM 1999. Spatial variation of woolly apple aphid (Eriosoma lanigerum, Hausmann) in a genetically diverse apple planting. New Zealand Journal of Ecology 23(1): 39-44.

Benge J, Barber A, McCusker K, Le Quellec I, Manhire J, Hunt L, Moller H, Rosin C, MacLeod CJ 2014. The New Zealand sustainability dashboard: connecting growers, industry, consumers, regulators and policy makers to drive sustainable horticulture. XXIX International Horticultural Congress on Horticulture: Sustaining Lives, Livelihoods and Landscapes (IHC2014). SHS Acta Horticulturae 1103: 69-74. https://doi.org/10.17660/ ActaHortic.2015.1103.11

Cambouris AN, Zebarth BJ, Ziadi N, Perron I 2014. Precision agriculture in potato production. Potato Research 57(34): 249-262. https://doi.org/10.1007/s11540-0149266-0

Campbell RE, Roy S, Curnow T, Walter M 2016. Monitoring methods and spatial patterns of European canker disease in commercial orchards. New Zealand Plant Protection 69: 213-220. https://doi.org/10.30843/ nzpp.2016.69.5883

Cozzens T. 2020 Australia, New Zealand commit to develop SBAS by 2023. GPS World. Accessed 16 June 2020 from https://www.gpsworld.com/australia-new-zealandcommit-to-develop-sbas-by-2023/

Di Iorio D 2019. Neonectria ditissima spatial pattern and progression in Tasman and Hawke's Bay, New 
Zealand. Thesis submitted in partial fulfilment of the requirements Master of Science degree, Farming Systems Ecology Group, Wageningen University, The Netherlands, $60 \mathrm{p}$.

Di Iorio D, Walter M, Lantinga E, Kerckhoffs H, Campbell RE 2019. Mapping European canker spatial pattern and disease progression in apples using GIS, Tasman, New Zealand. New Zealand Plant Protection 72: 176-184. https://doi.org/10.30843/nzpp.2019.72.305

ESRI 2020a. A quick tour of attribute domains. Accessed 16 June 2020 from https://desktop.arcgis.com/ en/arcmap/latest/manage-data/geodatabases/anoverview-of-attribute-domains.htm

ESRI 2020b. Overview of joins and relates. Accessed 16 June 2020 from https://pro.arcgis.com/en/pro-app/help/ data/tables/joins-and-relates.htm

Jordan EJ, Moran C, Godwyll JM 2019. Does tourism really cause stress? A natural experiment utilizing ArcGIS Survey123. Current Issues in Tourism. https://doi.org/ $10.1080 / 13683500.2019 .1702001$

Liu Y, Langemeier MR, Small IM, Joseph L, Fry WE 2017. Risk management strategies using precision agriculture technology to manage potato late blight. Agronomy Journal 109(2): 562-575. https://doi.org/10.2134/ agronj2016.07.0418

Luvisi A, Ampatzidis YG, De Bellis, L 2016. Plant pathology and information technology: opportunity for management of disease outbreak and applications in regulation frameworks. Sustainability 8: (831). https:// doi.org/10.3390/su8080831

Malek R, Tattoni C, Ciolli M, Corradini S, Andreis D, Ibrahim A, Mazzoni V, Eriksson A, Anfora G 2018. Coupling traditional monitoring and citizen science to disentangle the invasion of Halymorpha halys. International Journal of Geo-Information 7: (171). https://doi.org/10.3390/ ijgi7050171

Monmonier M 1996. How to Lie with Maps. MI, USA: University of Chicago Press. 256 p. https://doi. org/10.7208/chicago/9780226029009.001.0001

National Research Council USA 1997. Precision agriculture in the 21st century: Geospatial and information technologies in crop management. Washington, DC: The National Academies Press 168 p.

Oliver MA 2010. Geostatistical applications for precision agriculture [electronic resource]. Dordrecht, New York: Springer. $331 \mathrm{p}$.

Orozco-Fuentes S, Griffiths G, Holmes MJ, Ettelaie R, Smith J, Baggaley AW, Parker NG 2018. Early warning signals in plant disease outbreaks. Ecological Modelling 393: 1219. https://doi.org/10.1016/j.ecolmodel.2018.11.003

Padgitt S, Petrzelka P, Wintersteen W, Imerman E 2001. Integrated crop management: The other precision agriculture. American Journal of Alternative Agriculture 16(1): 16-22. https://doi.org/10.1017/ $\underline{\text { S088918930000881X }}$

Rodriguez E, Garcia-Garrido JM, Garcia PA, Campos M 2009. Large-scale epidemiological study and spatial patterns of Verticillium wilt in olive orchards in southern Spain. Crop Protection 28: 46-52. https://doi.org/10.1016/j. cropro.2008.08.009
Santiago-Brown I, Metcalfe A, Jerram C, Collins C 2014. Transitional comparison of sustainability assessment programs for viticulture and a case-study on programs' engagement processes. Sustainability 6(4): 2031-2066. https://doi.org/10.3390/su6042031

Sayers RG, Sayers GP, Mee JF, Good M, Bermingham ML, Grant J, Dillon PG 2013. Implementing biosecurity measures on dairy farms in Ireland. The Veterinary Journal 197(2): 259-267. https://doi.org/10.1016/j.tvjl.2012.11.017

Srivastava DJ, Vosegaard T, Massiot D, Grandinetti PJ 2020. Core Scientific Dataset Model: A lightweight and portable model and file format for multi-dimensional scientific data. PLoSONE 15(1): e0225953. https://doi. org/10.1371/journal.pone.0225953

Van Rossum G \& Drake Jr FL 1995. Python reference manual. Centrum voor Wiskunde en Informatica Amsterdam.

Warkentine L 2020. Ecological and economic values of public trees from the Manitou tree inventory. Thesis Submitted in Partial Fulfilment of the Requirements for the Degree of Honours Bachelor of Environmental Management, Faculty of Natural Resources Management, Lakehead University, Thunder Bay, Ontario, $81 \mathrm{p}$.

Zhu X 2016. GIS for environmental applications: a practical approach. New York: Routledge, 471 p. https://doi. org/10.4324/9780203383124 